\title{
BMJ
}

\section{Designing prevention programmes to reduce incidence of dementia: prospective cohort study of modifiable risk factors}

\author{
K Ritchie, senior research director, ${ }^{1,2,3}$ I Carrière, research fellow, ${ }^{1,2} \mathrm{C}$ W Ritchie, consultant psychiatrist, ${ }^{3} \mathrm{C}$ Berr, \\ research director, ${ }^{1,2} \mathrm{~S}$ Artero, research fellow, ${ }^{1,2} \mathrm{M}$-L Ancelin, research director ${ }^{1,2}$
}

Inserm, U888 Nervous System Pathologies: Epidemiological and Clinical Research, La Colombière Hospital, 34093 Montpellier Cedex 5, France

${ }^{2}$ Université de Montpellier 1 , Montpellier, F-34000, France

${ }^{3}$ Faculty of Medicine, Imperial College, St Mary's Hospital, London

Correspondence to: K Ritchie karen.ritchie@inserm.fr

Cite this as: $B M J$ 2010;341:C3885 doi:10.1136/bmj.c3885

\section{ABSTRACT}

Objective To estimate the percentage reduction in incidence of dementia that would be obtained if specific risk factors were eliminated.

Design Prospective seven year cohort study.

Setting General population, Montpellier, France.

Participants 1433 people aged over 65 with a mean baseline age of 72.5 (SD 5.1) years.

Main outcome measures Diagnosis of mild cognitive impairment or dementia established by a standardised neurological examination.

Results Cox models were constructed to derive hazard ratios and determine confounding and interaction effects for potentially modifiable risk factors for dementia. Mean percentage population attributable fractions were calculated with $95 \%$ confidence intervals derived from bootstrapping for seven year incidence of mild cognitive impairment or dementia. The final model retained crystallised intelligence (population attributable fraction $18.11 \%$, 95\% confidence interval $10.91 \%$ to $25.42 \%$ ), depression (10.31\%, 3.66\% to $17.17 \%$ ), fruit and vegetable consumption $(6.46 \%, 0.15 \%$ to $13.06 \%)$, diabetes (4.88\%, $1.87 \%$ to $7.98 \%$ ), and apolipoprotein E $\varepsilon 4$ allele (7.11\%, $2.44 \%$ to $11.98 \%)$.

Conclusions Increasing crystallised intelligence and fruit and vegetable consumption and eliminating depression and diabetes are likely to have the biggest impact on reducing the incidence of dementia, outweighing even the effect of removing the principal known genetic risk factor. Although causal relations cannot be concluded with certainty, the study suggests priorities that may inform public health programmes.

\section{INTRODUCTION}

The past 30 years have seen the proliferation of meth odologically well constructed prospective population studies of dementia that have pointed to multiple risk factors. Although the exact cause of the dementias remains unknown, these studies together suggest a complex interaction of exposures that contribute differentially to the probability and timing of onset of disease. The contribution of epidemiology to dementia research has, however, been principally in the areas of observational and analytical epidemiology-that is, the establishment of incidence and prevalence rates and demonstration of interactive risk factors used to inform clinical research and drug treatment. On the other hand, interventional epidemiologythat is, the designation of intervention points at the level of the population for the reduction of disease incidence- has been relatively neglected. Historically, this has been an important function of epidemiological research; public health strategies based on risk removal have previously reduced disease incidence even when the exact cause of the disease was still unknown-for example, hand washing to prevent puerperal fever, controlling water sources to prevent cholera, and recommending the use of condoms to prevent the spread of AIDS.

Clinical and biological research into the dementias had made considerable progress towards the end of the 20th century, leading to the marketing of treatments for the symptoms of dementia that will most likely, however, have the effect of increasing the prevalence of disease by extending its duration without having an impact on incidence. In the face of a pandemic of dementia, with predicted increases of $100 \%$ in developed countries between 2001 and 2020, and of 300\% in China, India, and neighbouring south Asian and western Pacific countries, ${ }^{1}$ even small reductions in incidence, or delaying the age of onset, are likely to have significant effects on prevalence and the enormous associated public health burden. The application of a general population approach to prevention of dementia based on the principles of interventional epidemiology, and targeting risk factors likely to decrease incidence in the absence of a coherent aetiological model, thus seems timely.

This study firstly identified candidate risk factors for dementia that have already been identified by population studies using internationally recognised algorithms for dementia, and which may potentially be the focus of intervention strategies. The second step was to examine within a prospective cohort the impact on incidence of the theoretical elimination of each of these risk factors, taking into account the other factors. 
The aim of the study was to determine, on the basis of current epidemiological knowledge, which interventions at a population level might be most efficient in reducing the burden of dementia in the coming years if no effective treatment is found.

\section{METHODS}

\section{Candidate exposures}

We identified potentially modifiable risk factors for dementia from population and clinical studies cited in PubMed since 1980 (this date coincides with the publication of internationally accepted research guidelines for diagnosis of dementia). These candidate intervention factors may broadly be divided into sociodemographic, clinical, and environmental and lifestyle factors.

Sociodemographic factors-Age is the most important risk factor for dementia; the incidence rises exponentially between the ages of 60 and 80 , and the rate of increase slows thereafter. ${ }^{2-4}$ Although theoretically non-modifiable, this is a principal confounding factor that must be taken into account. Education seems to be an important protective factor; evidence indicates that this may be due to compensation in higher age groups (neuronal reserve), which delays the onset of clinical signs. ${ }^{5}$ Ethnic differences consist of both non-modifiable (genetic) and modifiable (cultural) components. Most ethnic differences in risk for dementia that have been identified are due to methodological differences, with the exception of a study of Africans in Nigeria and the United States, in which the second group were found to have a higher incidence of dementia, ${ }^{6}$ suggesting a modifiable component.

Clinical factors - Risk for late onset Alzheimer's disease is known to be associated with polymorphisms of the apolipoprotein $\mathrm{E}$ (ApoE) gene; people with an $\varepsilon 4$ allele have an increased risk of both familial and sporadic forms, accounting for $20-50 \%$ of the attributable risk. ${ }^{78}$ Although this is currently a non-modifiable risk factor, we cite it here because of its potential for modifying the impact of other factors, implying that some interventions may perhaps best be restricted to people at genetic risk. With regard to past health events, head injury has been found to double the risk for Alzheimer's disease in men, ${ }^{9}$ with increased risk in those with an ApoE $\varepsilon 4$ allele. ${ }^{10}$ Established vascular risk factors (heart disease, stroke, hypertension, obesity, diabetes, and elevated plasma homocysteine and cholesterol concentrations) increase the risk for both vascular dementia and Alzheimer's disease and accelerate the associated cognitive decline. ${ }^{11-16} \mathrm{~A}$ history of depression, asthma, and repeated exposure to herpes simplex type I virus in the presence of an ApoE $\varepsilon 4$ allele have also been observed to increase the risk of dementia. ${ }^{17-19}$ With regard to treatments, the use of non-steroidal anti-inflammatory agents and hormonal replacement therapy in menopausal women has been found by most studies to reduce the risk for dementia and also to diminish the risk effect of ApoE $\varepsilon 4,{ }^{20-22}$ whereas exposure to anaesthesia and use of drugs with anticholinergic effects have been shown to increase risk. ${ }^{23-25}$

Environmental and lifestyle factors -Participation in cognitively stimulating activities and an active and socially integrated lifestyle have both been observed to delay the onset of dementia. ${ }^{26-28}$ A Mediterraneantype diet with high consumption of vegetables and fish (n-3 polyunsaturated fatty acids) has been observed to reduce the risk of incident Alzheimer's disease. ${ }^{2930}$ Moderate consumption of alcohol has been observed to be a protective factor in non-ApoE $\varepsilon 4$ carriers, ${ }^{31} 32$ whereas heavy drinking may lower the age of onset of Alzheimer's disease. ${ }^{33}$ Caffeine consumption has been associated with a reduction in density of white matter lesions and possible delaying of onset of dementia, ${ }^{34}$ and inadequate consumption of water has been observed to increase risk. ${ }^{35}$ Nicotine intake has also been observed to decrease the risk of dementia, ${ }^{36}$ although more recent studies have suggested that it may increase it and also bring forward age of onset with an ApoE $\varepsilon 4$ interactive effect. ${ }^{37}$

Not all of the above risk factors have been consistently confirmed by subsequent studies, and considerable inconsistencies exist between observations as a result of methodological differences. We do not intend the above summary to critically review these studies, however, but rather to generate a list of candidate risk factors for which strong empirical evidence already exists and which are modifiable, for use in modelling the effects of interventions. The risk factors considered here are those that may potentially be avoided or reversed, with the exception of ApoE \&4 genotype, which we have retained as a benchmark for comparison purposes given its strong and consistent association with dementia and cognitive decline. We included age and sex as adjustment variables.

\section{Participants}

The Esprit Study is a neuropsychiatric cohort study of community dwelling people aged 65 years and over drawn at random from the electoral rolls of Montpellier in the south of France and recruited between 1999 and 2001. Participants have been reassessed at baseline and at two, four, and seven years. Each participant gave written informed consent.

\section{Procedures}

A neurologist examined all participants at baseline and at two, four, and seven year follow-up by using a standardised interview incorporating cognitive testing to identify both dementia and mild cognitive impairment. ${ }^{38}$ Mild cognitive impairment is considered to be a prodrome of dementia; we have included it here to cover cases of slowly evolving dementia that may be diagnosed after the seven year follow-up. We have previously shown that the application of the modified Peterson's criteria for mild cognitive impairment in this population identifies people with cognitive difficulties with a very high likelihood of later developing dementia. ${ }^{38}$ These participants cannot be considered not to have dementia, and their removal would risk 
biasing results. We therefore make the assumption that a public health intervention should target both diagnosed and prodromal dementia, and we included incident cases of mild cognitive impairment with dementia in our analyses. We defined people with mild cognitive impairment as those with a score (on at least one cognitive test) in the lowest baseline fifth in relation to the relevant age matched and education matched group and with a cognitive complaint. We used fifths rather than standard deviations, as in other studies in which the cognitive scores are not normally distributed. A panel of expert neurologists further validated all incident cases of dementia and mild cognitive impairment independently from the study investigators. We considered the date of onset of dementia or mild cognitive impairment to be the date of the follow-up interview at which dementia or mild cognitive impairment was diagnosed.

A standardised interview by trained nurses included questions on sociodemographic characteristics and educational level (classified in four groups corresponding to $5,9,12$, and $\geq 12$ years of education). The Neale adult reading test is a measure of IQ that is based on degree of exposure to, and integration of, culture specific information and is usually not modified in early stage dementia. ${ }^{39}$ It may be considered to be a measure of crystallised intelligence. We chose the threshold for the reading test score $(<20$ corresponding to lower performance) in view of the non-linear risk curve (test of non-linearity, $\mathrm{P}=0.008$ ). We used this measure as a proxy for lifelong cognitive activity as a complement to years of education, which covers principally childhood. We also obtained information relating to monthly income, mobility and confinement to home and neighbourhood, height, and weight. We used nutritional questionnaires to obtain information on consumption of fruit and vegetables (two portions a day versus less) and fish (once a week versus less). We obtained information on type and quantity of alcohol consumption $>36 \mathrm{~g} /$ day in men and $>24 \mathrm{~g} /$ day in women versus less) and tobacco use (classified as past/present or never users), daily water consumption (less than 11 /day versus $\geq 1$ 1/day), and coffee and tea intake ( $\leq 3 \mathrm{cups} /$ day versus more, assuming one cup of coffee to be equal to two cups of tea).

We established history of exposure to anaesthesia over two years; repeated herpes infections; asthma; and lifetime history of hypertension, hypercholesterolaemia, stroke, and ischaemic heart disease (angina pectoris, myocardial infarction, cardiac and vascular surgery) according to standardised questions with additional information where necessary from general practitioners. For people who reported the occurrence of vascular events during follow-up, we obtained confirmation from general practitioners, specialists, and hospital records. The interview also included an inventory of all drugs used during the preceding month, noting those with potential anticholinergic effects, ${ }^{2425}$ and past and present versus never users of hormone replacement therapy. The interviewer saw medical prescriptions and, where feasible, the drugs themselves.
We assessed depressive symptoms by using the Center for Epidemiological Studies depression scale with a threshold of 16 points, ${ }^{40}$ and we diagnosed current major depressive episodes with the MINI neuropsychiatric examination. ${ }^{41}$ We considered participants identified by either method or taking antidepressants to be cases of depression. We measured blood pressure twice during the interview by using a digital electronic tensiometer OMRON M4, with hypertension defined as the mean of the two measures being $\geq 140 / 90 \mathrm{~mm}$ $\mathrm{Hg}$ or treatment for hypertension and high pulse rate as $\geq 80 \mathrm{bpm}$. We took fasting blood samples for ApoE $\varepsilon 4$ genotyping and assessment of hypercholesterolaemia (high total cholesterol $\geq 7.25 \mathrm{mmol} / \mathrm{l}$ or use of cholesterol lowering agents) and diabetes (defined as glycaemia $\geq 7 \mathrm{mmol} / 1$ or antidiabetic treatment). We dichotomised body mass index (weight in kilograms divided by the square of height in metres) as lower than 20.5 versus 20.5 or above. We defined baseline metabolic syndrome according to the National Cholesterol Education Program Adult Treatment Panel III criteria, which require the presence of three or more alterations among the following cardiometabolic parameters: high systolic $(>130 \mathrm{~mm} \mathrm{Hg}$ ) or diastolic blood pressure $(>85 \mathrm{~mm} \mathrm{Hg})$ or use of antihypertensive drugs; large waist circumference $>88 \mathrm{~cm}$ in women and $>102 \mathrm{~cm}$ in men); high triglycerides $(\geq 1.7 \mathrm{mmol} /$ 1); low high density lipoprotein cholesterol (men $<1.04$ and women $<1.29 \mathrm{mmol} / \mathrm{l}$ ); and high fasting glycaemia $(\geq 6.1 \mathrm{mmol} / \mathrm{l})$ or antidiabetes drugs. ${ }^{42}$

Although the type of dementia was differentiated in the clinical interview, we have chosen to group together all dementias and mild cognitive impairment. Differentiation may be preferable in an analytical study attempting to clarify causation, but in this context the principal outcome will be a public health intervention at a population level, which we assume would preferably target all dementia and its prodrome state. Moreover, most of the risk factors examined are common to the different forms of dementia.

\section{Statistical methods}

We used a prospective general population database (the Esprit Study) to examine the individual impact of each of these risk factors and protective factors on the incidence of dementia by calculating hazard ratios. We used a Cox model with delayed entry in the longitudinal analysis of incident cases of mild cognitive impairment or dementia over the seven year follow-up, taking age as the basic timescale and birth as the time origin. We censored participants who died without dementia or mild cognitive impairment at their age of death and those who were lost to follow-up before developing dementia or mild cognitive impairment at the last cognitive examination. The age of onset of the event (dementia or mild cognitive impairment) was the middle of the interval between the last follow-up without the event and the first follow-up with the event. The risk factors found to be significant $(\mathrm{P}<0.15)$ in a univariate model (adjusted for sex) were included in the multivariate model, which was reduced with a 
backward step by step method. We used SAS (v9.1) for the statistical analyses.

Given that many of the exposures discussed above are likely to be relatively rare, so intervention at a general population level may not be cost effective, the population attributable fraction is a better index of the potential for prevention through general population intervention than is attributable risk. The population attributable fraction takes into account both the relative risk and the frequency of the risk factor; it estimates the proportion of incident cases in the general population that would be prevented if the exposure was entirely eliminated. The population attributable fraction theoretically estimates the reduction in incidence of disease that would be obtained if the entire population was not exposed to a given risk, compared with current rates of exposure. However, as the calculations are based on incidences, a time of reference must be taken into account (incidence over a given number of years $)^{43}$; furthermore, in the case of diseases such as dementia with multifactorial aetiology, competing risk factors must also be simultaneously considered. The definition of population attributable fraction used here is thus the mean proportion in risk reduction over seven years that would be obtained if a specific exposure was entirely eliminated from the population while the distribution of other risk factors remains unchanged. ${ }^{44}$

Estimation of population attributable fraction requires categorical covariates (preferably binary to avoid ambiguity in interpretation), so a threshold must be selected that is both clinically meaningful and pragmatic as a potential public health intervention. As with any multivariate model, problems of colinearity arise from the simultaneous use of highly correlated variables. Interaction effects also need to be tested to determine whether results can be pooled. In the case of dementia, we need to consider, for example, whether separate calculations should be made for men and women or for people with and without an $\mathrm{ApoE} \varepsilon 4$ allele; the corollary is that specific intervention programmes should then be developed for these subgroups.

We initially included all of the risk factors described above in our analyses (with the exception of age and sex, which are considered non-modifiable and appear as adjustment variables, and ethnicity, which could not be included in this French study for ethical reasons). Covariates have been selected by Cox models including all risk and confounding factors. The model is also used to test interaction effects. The formula most commonly cited for the calculation of population attributable fraction is $P A F=I R_{\text {total exposed and non-exposed }}-I_{\text {non-exposed }}$ IRtotal exposed and non-exposed, where IR=incidence risk.

However, this model is not appropriate in the case of the dementias, for which interaction and confounding across variables are likely. We have thus used a formula based on the adjusted relative risks $\left(\mathrm{RR}_{\mathrm{adj}}\right)$ derived from a multivariate model: $\mathrm{PAF}=\left\{\left(\mathrm{RR}_{\mathrm{adj}}-1\right)\right\}$ $\left.\mathrm{RR}_{\mathrm{adj}}\right\} \mathrm{P}_{\mathrm{e}(\text { (cases) }}$, where $\mathrm{P}_{\mathrm{e}(\text { cases })}$ is the prevalence of the exposure in participants with dementia. We calculated the relative risk with a Cox model yielding hazard ratios. The confidence intervals for the population attributable fraction are estimated by the bootstrap re-sampling method. In our analysis, we generated 1000 samples from the original dataset, calculated the population attributable fractions for each sample, and calculated the 95\% confidence interval.

\section{RESULTS}

For our analyses, we used data from the 1433 people without a diagnosis of either mild cognitive impairment or dementia at baseline, for whom cognitive status was assessed during follow-up and data were available on all risk factors for the final model (figure). The baseline mean age of the population was 72.5 (SD 5.1) years. The median length of follow-up was 7.31 (interquartile range 2.72-7.58) years, and 405 incident cases of all cause dementia and mild cognitive impairment were validated. Table 1 shows the baseline sociodemographic and clinical characteristics of participants, along with a non-adjusted $\chi^{2}$ comparison of participants who developed mild cognitive impairment or dementia over the next seven years with those who did not for each of the potential risk factors. We found no difference between men and women in rates of incident mild cognitive impairment or dementia.

We examined the association between each of these candidate risk factors and incidence of mild cognitive impairment or dementia at two, four, or seven year follow-up by univariate analyses adjusted for age and sex. Results showed significant associations of incidence with low education and Neale adult reading test score, depression, stroke, diabetes, ApoE $\varepsilon 4$ allele, ischaemic heart disease, head injury, caffeine consumption, exposure to anaesthesia, and fruit and vegetable consumption. We found no interaction effects with sex and ApoE $\varepsilon 4$ allele, except an interaction between sex and depression $(\mathrm{P}=0.03$; depressed

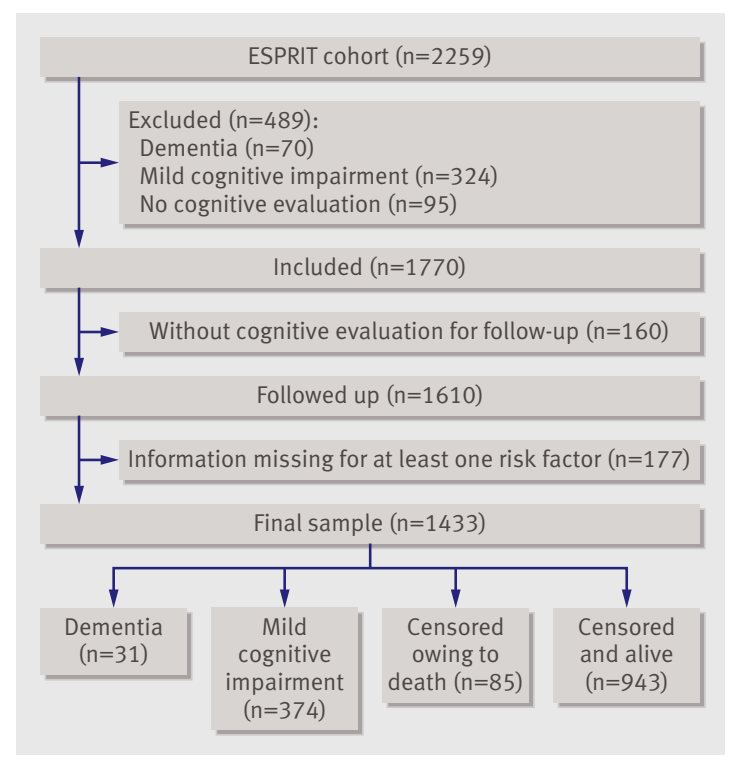

Flow diagram 
Table 1|Baseline characteristics of participants and sex and age adjusted hazard ratio for seven year incident mild cognitive impairment $(M C I)$ or dementia $(n=1433)$. Values are numbers (percentages) unless stated otherwise

\begin{tabular}{|c|c|c|c|c|c|}
\hline \multirow[b]{2}{*}{ Characteristic } & \multirow{2}{*}{$\begin{array}{l}\text { No dementia or } \mathrm{MCl} \text { during } \\
\quad \text { follow-up }(n=1028)\end{array}$} & \multirow{2}{*}{$\begin{array}{l}\text { Dementia or } \mathrm{MCl} \text { during } \\
\text { follow-up }(\mathrm{n}=405)\end{array}$} & \multirow{2}{*}{$\begin{array}{l}X^{2} \text { test } P \\
\text { value }\end{array}$} & \multicolumn{2}{|c|}{ Cox model } \\
\hline & & & & Hazard ratio $(95 \% \mathrm{Cl})$ & $P$ value \\
\hline Female & $621(60.4)$ & $241(59.5)$ & 0.75 & & \\
\hline \multicolumn{6}{|l|}{ Age (years): } \\
\hline $65-69$ & $397(38.6)$ & $85(21.0)$ & \multirow{4}{*}{$<0.001$} & & \\
\hline $70-74$ & $358(34.8)$ & $138(34.1)$ & & & \\
\hline $75-80$ & $194(18.9)$ & $117(28.9)$ & & & \\
\hline$\geq 80$ & $79(7.7)$ & $65(16.1)$ & & & \\
\hline Education $\leq 5$ years & $214(20.8)$ & $98(24.2)$ & 0.16 & 1.27 (1.01 to 1.59$)$ & 0.04 \\
\hline Neale adult reading test $<20$ & $311(30.3)$ & $176(43.5)$ & $<0.001$ & 1.74 (1.43 to 2.12$)$ & $<0.001$ \\
\hline Body mass index $<20.5$ & $87 / 1022(8.5)$ & $31 / 401(7.7)$ & 0.63 & 0.82 (0.56 to 1.19$)$ & 0.28 \\
\hline Diabetes & $57(5.5)$ & $43(10.6)$ & $<0.001$ & 1.93 (1.40 to 2.66$)$ & $<0.001$ \\
\hline Fish <once/week & $117 / 1027$ (11.4) & $50 / 405(12.4)$ & 0.61 & $1.13(0.84$ to 1.52$)$ & 0.43 \\
\hline Fruit/vegetables «twice/day & $256(24.9)$ & $127(31.4)$ & 0.01 & 1.38 (1.11 to 1.70$)$ & 0.003 \\
\hline Depression & $290(28.2)$ & $149(36.8)$ & 0.002 & 1.48 (1.20 to 1.82$)$ & $<0.001$ \\
\hline Hypertension & $571 / 967(59.1)$ & $220 / 370(59.5)$ & 0.89 & 0.96 (0.78 to 1.19$)$ & 0.72 \\
\hline Hypercholesterolaemia & $352 / 1027$ (34.3) & $139 / 405(34.3)$ & 0.99 & 1.06 (0.86 to 1.30$)$ & 0.61 \\
\hline Anticholinergic drugs & $42(4.1)$ & $23(5.7)$ & 0.19 & 1.41 (0.92 to 2.15$)$ & 0.11 \\
\hline Ischaemic heart disease & $91(8.9)$ & $50(12.3)$ & 0.05 & 1.39 (1.03 to 1.89 ) & 0.03 \\
\hline Stroke & $21 / 1020(2.1)$ & $15 / 401(3.7)$ & 0.07 & 1.96 (1.16 to 3.29$)$ & 0.01 \\
\hline Head injury & 79/1018 (7.8) & $37 / 400(9.3)$ & 0.36 & 1.46 (1.03 to 2.05$)$ & 0.03 \\
\hline Asthma & $20 / 1022(2.0)$ & $3 / 400(0.8)$ & 0.10 & 0.42 (0.13 to 1.30$)$ & 0.13 \\
\hline Metabolic syndrome & 132/1027 (12.9) & $53 / 405(13.1)$ & 0.91 & $1.10(0.82$ to 1.47$)$ & 0.52 \\
\hline Herpes & $375 / 1022$ (36.7) & $156 / 402(38.8)$ & 0.46 & 1.11 (0.91 to 1.35$)$ & 0.32 \\
\hline Living alone & 270/1025 (26.3) & $119 / 405(29.4)$ & 0.24 & 1.00 (0.79 to 1.27$)$ & 0.99 \\
\hline Caffeine $\leq 3$ cups/day & $792(77.0)$ & $338(83.5)$ & 0.007 & 1.37 (1.05 to 1.78$)$ & 0.02 \\
\hline Alcohol & $121 / 1006$ (12.0) & 45/399 (11.3) & 0.69 & $0.99(0.72$ to 1.37$)$ & 0.97 \\
\hline Current smoking & $63 / 1027$ (6.1) & $22 / 405(5.4)$ & 0.61 & $0.97(0.63$ to 1.49$)$ & 0.88 \\
\hline Water $<1 \mathrm{l} /$ day & $277 / 1027$ (27.0) & $104 / 405(25.7)$ & 0.62 & 0.91 (0.73 to 1.13$)$ & 0.39 \\
\hline Pulse rate $\geq 80 \mathrm{bpm}$ & 200/957 (20.9) & $62 / 369(16.8)$ & 0.09 & 0.81 (0.61 to 1.06$)$ & 0.13 \\
\hline ApoE4 ع4 allele & $181(17.6)$ & $90(22.2)$ & 0.04 & 1.41 (1.11 to 1.78$)$ & 0.005 \\
\hline Anaesthesia $0-2$ years & $301(29.3)$ & $145(35.8)$ & 0.02 & 1.28 (1.05 to 1.57$)$ & 0.02 \\
\hline
\end{tabular}

women at higher risk). The remaining analyses were therefore not stratified.

We constructed a global stepwise descending Cox model from risk factors in the previous analysis. We took age as the basic timescale and birth as the time origin in a delayed entry model (table 2 ). We included all the covariates found to be significant in the preceding step at a level of $\mathrm{P}<0.15$. This multivariate model was reduced by using a backward method with a probability of staying in the model of $\mathrm{P}<0.05$. The final model provides mean population attributable fraction estimations. We used multiple sampling (bootstrapping) to estimate 95\% confidence intervals. Crystallised intelligence (Neale adult reading test score), diabetes, ApoE4, fruit and vegetable consumption, and depression were retained in the final model. As Neale adult reading test scores and mild cognitive impairment are both indicators of poor cognitive performance, and the first is likely to be a risk factor for the second, we examined the risk of high and low reading test scores with incident dementia only as an outcome within a Cox model. This independent association remains strong (hazard ratio $2.9,95 \%$ confidence interval 1.6 to $5.2 ; \mathrm{P}<0.0005)$.

\section{DISCUSSION}

We constructed Cox models to derive hazard ratios and determine confounding and interaction effects for potentially modifiable risk factors for dementia and calculated mean percentage population attributable fractions for seven year incidence of mild cognitive impairment or dementia. The final model retained crystallised intelligence, depression, fruit and vegetable consumption, diabetes, and apolipoprotein E $\varepsilon 4$ allele.

\section{Relative impact of different risk factors}

Estimations of population attributable fraction suggest that elimination of the ApoE $\varepsilon 4$ allele from the general population would lead only to a $7.1 \%$ reduction in the number of incident cases of mild cognitive impairment or dementia over the next seven years. Genetic modification is clearly not a feasible goal for public health intervention, but as it constitutes one of the most significant risk factors for dementia we have taken it as a benchmark for the comparison of other exposures. The greatest impact in terms of prevention is estimated to come from increasing Neale adult reading test scores, leading to an $18.1 \%$ reduction in incidence of mild 


\begin{tabular}{|c|c|c|c|c|}
\hline \multirow[b]{2}{*}{ Risk factor } & \multicolumn{2}{|l|}{ Cox model ${ }^{*}$} & \multirow[b]{2}{*}{ \% PAF (95\% Cl†) } & \multirow{2}{*}{$\begin{array}{c}\text { Cumulative \% PAF } \\
(95 \% \mathrm{Cl} \dagger)\end{array}$} \\
\hline & Hazard ratio $(95 \% \mathrm{Cl})$ & $P$ value & & \\
\hline Neale adult reading test $<20$ & 1.72 (1.41 to 2.09 ) & $<0.001$ & 18.1 (10.9 to 25.4$)$ & 18.1 (10.9 to 25.4 ) \\
\hline Diabetes & 1.85 (1.34 to 2.56$)$ & $<0.002$ & $4.9(1.9$ to 8.0$)$ & \multirow{3}{*}{20.7 (11.2 to 29.8$)$} \\
\hline Depression & 1.39 (1.13 to 1.71$)$ & 0.002 & 10.3 (3.7 to 17.2$)$ & \\
\hline Fruit/vegetables «twice/day & $1.26(1.02$ to 1.56$)$ & 0.04 & $6.5(0.2$ to 13.1$)$ & \\
\hline ApoE4 $\varepsilon 4$ allele & $1.47(1.16$ to 1.86$)$ & 0.001 & $7.1(2.4$ to 12.0$)$ & 7.1 (2.4 to 12.0$)$ \\
\hline
\end{tabular}

cognitive impairment or dementia. The reading test score - an indicator of crystallised intelligence, lifetime intellectual activity, and cultural exposure - is seen to have a greater impact than educational level, which was not retained by the initial risk model. This may be because it is a more accurate assessment of acquired learning than number of years of formal education in this generation, of whom many were exposed to war conditions (second world war and Spanish and Algerian civil wars), which interrupted schooling and professional training. Crystallised intelligence may be considered a proxy marker for exposure to intellectual activity, and determining whether this may have been confounded by association with higher physical activity might have been useful. Unfortunately, no simple, independent unitary measure of physical activity was available; however, a recent analysis has shown that the cognitive component of overall activity rather than the physical aspect is protective in relation to onset of dementia. ${ }^{27}$ The Neale adult reading test score is a combination of both genetic ability and cultural exposure, but this analysis cannot distinguish which component is having the effect of reducing incidence. In the absence of an answer to this central question, the public health message can only be to encourage literacy at all ages irrespective of innate ability.

Eliminating depression and diabetes and increasing fruit and vegetable consumption were estimated to lead to an overall $20.7 \%$ reduction in incidence of dementia. Of these, depression makes the greatest contribution; elimination of depression from the elderly population was estimated to lead to a mean $10.3 \%$ reduction in the number of new cases over seven years. Our analyses included treatment for depression and diabetes. The underlying assumption was that although treatment may have eliminated symptoms and reduced risk through decreasing length of exposure, the person has nevertheless been exposed to all the underlying negative biological effects associated with the disease state that may be linked to the aetiology of dementia, such as inflammation, increased permeability of the blood-brain barrier, damage to white matter, and raised cortisol concentrations. In this context, the corollary of our population attributable fraction analyses in terms of public health interventions is that these disorders should not so much be treated as prevented from occurring at all. This would imply targeting and monitoring of people at high risk and introducing preventive treatment where feasible.

However, the causal relation between depression and dementia remains unclear. Depression may be a prodromal sign of dementia, such that its treatment even at an early stage may not prevent onset of dementia, although previous research has suggested that its elimination can at least slow the rate of functional loss. ${ }^{45}$ Alternatively, depression may be an independent contributor to risk through, for example, inflammatory processes or hippocampal damage via a glucocorticoid cascade, such that treatment may delay onset. Meta-analyses have failed to adequately resolve this question, ${ }^{17}$ and an intervention programme aiming at preventing prolonged exposure to depression by early treatment (especially in family members of people with dementia) would greatly increase our current knowledge of this association.

\section{Comparison with other studies}

Recent papers reviewing promising strategies for prevention of dementia have underlined the importance of controlling vascular risk factors, mental activity, and depression, but have not been able to prioritise interventions or take into account exposure rates. ${ }^{4647} \mathrm{Clin}$ ical research in recent years has focused principally on hypertension as an intervention measure; however, the HYVET-COG randomised controlled trial of prevention of hypertension actually found a lower two year incidence of dementia in the placebo group. ${ }^{48}$ Our analyses also suggest significant benefit not from elimination of hypertension alone but rather its associated risk factor diabetes. The recent study of Li et al further supports this finding, reporting significant reductions in the incidence of dementia among users of angiotensin receptor blockers, ${ }^{49}$ which offer increased protection against diabetes and stroke compared with other antihypertensive agents. However, given that the followup period in our study is shorter than that of some previous studies of hypertension, we may have underestimated the importance of this factor.

\section{Potential for intervention}

The general conclusion from our population attributable fraction analyses is that, in the continued absence of an effective treatment for the dementias, public health programmes should aim above all at prevention of diabetes, which is a well established risk factor for dementia, is unlikely to be simply a dementia prodrome, is highly prevalent, and is treatable. The high prevalence of this disorder in the elderly population means that intervention is likely to have a high impact and be cost effective, even though the relative risk is modest. Although increased fruit and vegetable consumption and crystallised intelligence also have high potential impact, their relation to dementia, and the intervening role of lifestyle factors, makes it difficult to formulate strategies for health intervention at a population level. Depression is also common, is relatively easy to treat, and frequently precedes onset of dementia; however, its status as a risk factor rather than (or as well as) an early 
feature of the dementia itself is not clear. Clinical levels of depression are, however, relatively easy to screen at a population level and should be treated whether or not they are a prodromal feature of dementia; this will in any case diminish the rate of functional loss.

The most reasonable way forward, taking into account the above considerations, would be the construction of an intervention programme within the general population aimed at early screening for glucose tolerance and insulin resistance and early treatment of raised depressive symptoms. These risk factors have been highlighted by previous epidemiological research, but this is the first study of comparative benefit, estimating the relative advantages of elimination of various risk factors. These factors are not necessarily the most important in terms of disease causality, but rather those whose elimination, by virtue of their frequency as well as their risk level, is likely to have the greatest impact in reducing the number of future cases.

This leads to the final question of the age at which such interventions should be made. Given that prevention rather than treatment of these chronic states is to be the focus of intervention, this would imply targeting a younger age group. Empirical evidence would seem to support this; for example, dementia is linked to diabetes and early changes in cerebrovascular vessels in mid-life.$^{5051}$ A great limitation of current epidemiological and clinical research on dementia has been its focus on cohorts over the age of 65 , so that reliably estimating the extent of exposure to subclinical states before the onset of disorders such as depression and diabetes or determining the age at which intervention and prevention are most likely to be maximally effective has been impossible. Does controlling insulin resistance or eliminating depression have the same impact on incidence if it is carried out at 40 and 70 years?

\section{Strengths and limitations}

The main strength of our analyses is the use of a prospective database with diagnoses of dementia validated by neurologists, and not just according to a diagnostic algorithm, and also the inclusion of measures of almost all known modifiable risk factors. To our knowledge, this is the first attempt to model the effects of a theoretical population-wide prevention strategy which compares the relative effect of the removal of significant exposures.

The study is, however, open to several criticisms. We have made many decisions about level of exposure (for example biological thresholds and duration of symptoms), which may influence both hazard ratios and population attributable fraction calculations. Dimensional variables have been treated as binary, as required for population attributable fraction calculations, thus precluding the formulation of more precise clinical directives at an individual level but facilitating comparisons across cohorts. Although the calculation of potential impact fractions would have permitted the use of multiple categories and not just binary high and low exposure with these variables, ${ }^{52}$ the method assumes linearity, which we observed to not be the case. The use of potential impact fractions is also not considered appropriate when empirical data are available.

Although our model has examined individual risk factors while adjusting for all the others, we do not know, for example, to what extent they are inter-dependent in the real world such that totally eliminating one might modulate the appearance of others owing to other factors not accounted for here. Our findings should thus be validated in other datasets, using alternative definitions of exposure, before the construction of a public health intervention programme is considered. We have also made a decision to include mild cognitive impairment with dementia, although some of these people may not ever develop dementia. This seemed preferable to classifying them with the participants without dementia, given the very high risk of dementia in this group; retrospectively, this seems to have been the right decision given that only $7.6 \%$ of participants who developed full dementia in our study did not pass through a phase of mild cognitive impairment, thus confirming its status as a prodrome.

Our principal aim has been to show that the application of population attributable fractions to epidemiological studies of dementia may provide more meaningful directions for future public health initiatives than are currently used. However, the effectiveness of neutralising risk factors rests on the extent of the causal relation between the risk factor and onset of dementia. The risk factors included in this study have all been associated with credible underlying causative hypotheses, and confounding has been taken into account for a large number of factors, but the effect of their elimination cannot be precisely estimated in the absence of this information.

\section{Conclusions and policy implications}

The estimation of mean percentage population attributable fractions from a longitudinal study of risk factors for dementia suggests that reduction in the incidence of dementia over the next seven years would be maximised by the elimination of diabetes and possibly also depression (on the assumption that causality can be established such that it is clearly shown to be a risk factor and not just a disease prodrome). Increasing crystallised intelligence and consumption of fruit and vegetables also seem to have a potentially high impact at a population level but are difficult to implement as health prevention targets, as we cannot determine at which level of exposure they provide protective effects, and their relation to dementia is not easily differentiated from those of other lifestyle factors. Diabetes, or perhaps more specifically insulin resistance, stands for the moment as the primary target given that causality has been more clearly established than for the other factors highlighted in this analysis. Population attributable fraction calculations can provide only a crude estimate of impact on incidence, but they make a significant statement about public health priorities in disease prevention in the face of current knowledge. Epidemiological intervention studies in cohorts 


\section{WHAT IS ALREADY KNOWN ON THIS TOPIC}

Multiple potentially modifiable clinical and environmental risk factors for dementia have been identified from population studies

\section{WHAT THIS STUDY ADDS}

The relative impact on the incidence of dementia and mild cognitive impairment of removing different risk factors provides a hierarchy of priorities for public health interventions

Removing diabetes and depression along with increasing crystallised intelligence and fruit and vegetable consumption seem to have a greater impact than modifying genetic risk

Diabetes, and perhaps also depression, should be the principal targets of future population based health prevention programmes on; a partial replication. J Neurol Neurosurg Psychiatry 2003;74:857-62.

10 Mayeux R, Ottman R, Maestre G, Ngai C, Tang MX, Ginsberg H, et al. Synergistic effects of traumatic head injury and apolipoprotein epsilon 4 in patients with Alzheimer's disease. Neurology 1995;45:555-7.

11 Peila R, White LR, Petrovich H, Masaki K, Ross GW, Havlik RJ, et al. Joint effect of the APOE gene and midlife systolic blood pressure on late-life cognitive impairment: the Honolulu-Asia aging study. Stroke 2001;32:2882-9.

12 Razay G, Williams J, King E, Smith AD, Wilcock G. Blood pressure, dementia and Alzheimer's disease: the OPTIMA longitudinal study. Dement Geriatr Cogn Disord 2009;28:70-4.

13 Stewart R, Asonganyi B, Sherwood R. Plasma homocysteine and cognitive impairment in an older British African-Caribbean population. J Am Geriatr Soc 2002;50:1227-32.

14 White L, Launer L. Relevance of cardiovascular risk factors and ischemic cerebrovascular disease to the pathogenesis of Alzheime disease: a review of accrued findings from the Honolulu-Asia Aging Study. Alzheimer Dis Assoc Disord 2006;20:79-83S.

including younger adults are clearly needed to test the impact of intervention measures.

Contributors: M-LA and KR are the principal co-investigators of the Esprit Project. CB directed data collection. KR proposed the analysis, and the methods were developed by KR, M-LA, SA, and IC. IC did the analyses. KR wrote the article. KR, M-LA, CWR, and IC participated equally in the development of the discussion section. KR and IC are joint first authors. All authors read and commented on the final manuscript. Guilhem de Roquefeuil did the population sampling procedure for the Esprit Project. $\mathrm{KR}$ is the guarantor.

Funding: The Esprit Study was funded by an unconditional grant from Novartis and from the National Research Agency (ANR Project 07 LVIE004). Funding from Novartis was used to purchase equipment and pay for secretarial assistance. ANR funding provided salaries for the interviewers and the costs of clinical and biological examinations. Neithe body was involved in the statistical analyses or preparation of the manuscript.

Competing interests: All authors have completed the Unified Competing Interest form at www.icmje.org/coi disclosure.pdf and declare that (1) KR IC, CWR, CB, SA, and M-LA have technical support from Novartis for the submitted work; (2) KR, IC, CWR, CB, SA, and M-LA have no relationships with companies that might have an interest in the submitted work in the previous 3 years; (3) their spouses, partners, or children have no specified financial relationships that may be relevant to the submitted work; and (4) KR, IC, CWR, CB, SA, and M-LA have no non-financial interests that may be relevant to the submitted work.

Ethical approval: The ethics committee of the University-Hospital of Kremlin-Bicêtre approved the study protocol, and each participant gave written informed consent.

Data sharing: No additional data available.

1 Ferri C, Prince M, Brayne C, Brodaty H, Fratiglioni L, Ganguli M, et al. Global prevalence of dementia: a Delphi consensus study. Lancet 2005;17:2112-7.

2 Gao S, Hendrie HC, Hall KS, Hui S. The relationship between age, sex and the incidence of dementia and Alzheimer disease: a metaanalysis. Arch Gen Psychiatry 1998;55:809-15.

3 Lautenschlager NT, Cupples LA, Rao VS, Auerbach SA, Becker R, Burke J, et al. Risk of dementia among relatives of Alzheimer's disease patients in the MIRAGE study: what is in store for the oldest old? Neurology 1996;46:641-50.

4 Ritchie K, Kildea D. Is senile dementia age-related or ageing-related? Evidence from a meta-analysis of dementia prevalence in the oldest old. Lancet 1995;346:931-4.

5 Stern Y, Alexander GE, Prohovnik I, Mayeux R. Inverse relationship between education and parietotemporal perfusion deficit in Alzheimer's disease. Ann Neurol 1992;32:371-5.

6 Hendrie HC, Ogunniyi A, Hall KS, Baiyewu O, Unverzagt FW, Gureje O, et al. Incidence of dementia and Alzheimer disease in two communities: Yoruba residing in Ibadan and African Americans residing in Indianapolis, Indiana. JAMA 2001;14:739-47.

7 Seripa D, Panza F, Franceschi M, D’Onofrio G, Solfrizzi V, Dallapiccola B, et al. Non-apolipoprotein E and apolipoprotein E genetics of sporadic Alzheimer's disease. Ageing Res Rev 2009:8:214-36.

8 Ashford JW. APOE genotype effects on Alzheimer's disease onset and epidemiology. J Mol Neurosci 2004;23:157-65.

9 Fleminger S, Oliver DL, Lovestone S, Rabe-Hesketh S, Giora A. Head injury as a risk factor for Alzheimer's disease: the evidence ten years
15 Farooki A. Central obesity and increased risk of dementia more than three decades later. Neurology 2009;72:1030-1.

16 Helzner EP, Luchsinger JA, Scarmeas N, Cosentino S, Brickman AM, Glymour MM, et al. Contribution of vascular risk factors to the progression in Alzheimer disease. Arch Neurol 2009;66:343-8.

17 Jorm AF. History of depression as a risk factor for dementia: an updated review. Aust N Z J Psychiatry 2001;35:776-81.

18 Eriksson UK, Gatz M, Dickman PW, Fratiglioni L, Pedersen NL. Asthma, eczema, rhinitis and the risk for dementia. Dement Geriatr Cogn Disord 2008;25:148-56.

19 Itzhaki RF, Wozniak MA, Appelt DM, Balin BJ. Infiltration of the brain by pathogens causes Alzheimer's disease. Neurobiol Aging 2004;25:619-27.

20 Etminan M, Gill S, Samii A. Effect of non-steroidal anti-inflammator drugs on risk of Alzheimer's disease: systematic review and metaanalysis of observational studies. BMJ 2003;327:128.

21 Ancelin ML, Ritchie K. Lifelong endocrine fluctuations and related cognitive disorders. Curr Pharm Des 2005;11:4229-52.

22 Ryan J, Carriere I, Scali J, Dartigues JF, Tzourio C, Poncet M, et al. Characteristics of hormone therapy, cognitive function, and dementia: the prospective 3C Study. Neurology 2009;73:1729-37.

23 Baranov D, Bickler P, Crosby G, Culley DJ, Eckenhoff MF, Eckenhoff RG, et al. Consensus statement: first international workshop on anesthetics and Alzheimer's disease. Anesth and Analg 2009;108:1627-30.

24 Ancelin ML, Artero S, Portet F, Dupuy AM, Touchon J, Ritchie K. Nondegenerative mild cognitive impairment in elderly people and use of anticholinergic drugs: longitudinal cohort study. BMJ 2006;332:455-9.

25 Carriere I, Fourrier-Reglat A, Dartigues JF, Rouaud O, Pasquier F, Ritchie K, et al. Drugs with anticholinergic properties, cognitive decline, and dementia in an elderly general population: the 3-city study. Arch Intern Med 2009:169:1317-24.

26 Hall CB, Lipton RB, Sliwinski M, Katz MJ, Derby CA, Verghese J. Cognitive activities delay onset of memory decline in persons who develop dementia. Neurology 2009;73:356-61.

27 Akbaraly TN, Portet F, Fustinoni S, Dartigues JF, Artero S, Rouaud O, et al. Leisure activities and the risk of dementia in the elderly: results from the Three-City Study. Neurology 2009;73:854-61.

28 Fratiglioni L, Paillard-Borg S, Winblad B. An active and socially integrated lifestyle in late life might protect against dementia. Lancet Neurol 2004;3:343-53.

29 Scarmeas N, Luchsinger JA, Schupf N, Brickman AM, Cosentino S, Tang MX, et al. Physical activity, diet, and risk of Alzheimer disease. JAMA 2009;302:627-37.

30 Morris MC, Evans DA, Bienias JL, Tangney CC, Bennett DA, Wilson RS, et al. Consumption of fish and n-3 fatty acids and risk of incident Alzheimer disease. Arch Neurol 2003;60:940-6

31 Anstey KJ, Mack HA, Cherbuin N. Alcohol consumption as a risk factor for dementia and cognitive decline: meta-analysis of prospective studies. Am J Geriatr Psychiatry 2009;17:542-55.

32 Luchsinger JA, Tang MX, Siddiqui M, Shea S, Mayeux R. Alcohol intake and risk of dementia. J Am Geriatr Soc 2004;52:540-6.

33 Harwood DG, Kalechstein A, Barker WW, Strauman S, St George-Hyslop P, Iglesias C, et al. The effect of alcohol and tobacco consumption, and apolipoprotein $\mathrm{E}$ genotype, on the age of onset in Alzheimer's disease. Int J Geriatr Psychiatry 2010;25:511-8.

34 Ritchie K, Artero S, Portet F, Brickman A, Muraskin J, Beanino E, et al. Caffeine, cognitive functioning and white matter lesions in the elderly: establishing causality from epidemiological evidence. $J$ Alzheimer's Dis 2010;20(suppl 1):S161-6.

35 Gharibzadeh S, Hoseini SS. Chronic dehydration may be a preventable risk factor for Alzheimer's disease. Med Hypotheses 2007;68:718 
36 Van Duijn CM, Hofman A. Relation between nicotine intake and Alzheimer's disease. BMJ 1991;22:1491-4.

37 Lee Y, Back JH, Kim J, Kim SH, Na DL, Cheong HK, et al. Systematic review of health behavioral risks and cognitive health in older adults. Int Psychogeriatr 2009;9:1-14.

38 Artero S, Petersen R, Touchon J, Ritchie K. Revised criteria for mild cognitive impairment: validation within a longitudinal population study. Dement Geriatr Cogn Disord 2006;22:465-70.

39 Blair JR, Spreen O. Predicting premorbid IQ: a revision of the National Adult Reading Test. Clin Neuropsychol 1989;3:129-36.

40 Radloff L. The CES-D scale: a self-report depression scale for research in the general population. Appl Psychol Measurement 1977; 1:385-401.

41 Lecrubier Y, Sheehan D, Weiller E, Amorim P, Janavs J, Weiller E, et al. The Mini International Neuropsychiatric Interview (MINI), a short diagnostic interview: reliability and validity according to the CIDI. European Psychiatry 1997;12:232-41.

42 Grundy SM, Cleeman JI, Daniels SR, Donato KA, Eckel RH, Franklin BA et al. Diagnosis and management of the metabolic syndrome: an American Heart Association/National Heart, Lung, and Blood Institute scientific statement. Circulation 2005;112:2735-52.

43 Steenland K, Armstrong B. An overview of methods for calculating the burden of disease due to specific risk factors. Epidemiology 2006;17:512-9.

44 Rockhill B, Newman B, Weinberg C. Use and misuse of population attributable fractions. Am / Public Health 1998;88:15-9.

45 Ritchie K, Gilham C, Ledesert B, Touchon J, Kotzki PO. Depressive illness, depressive symptomatology and regional cerebral blood flow in elderly people with sub-clinical cognitive impairment. Age Ageing 1999;28:385-91.

46 Middleton L, Yaffe K. Promising strategies for the prevention of dementia. Arch Neurol 2009;66:1210-5.

47 Gammanpila UP, Burns A, Heller R, Purandare N. What are the benefits of cognitive enhancers for Alzheimer's disease: use of population impact measures. BMC Geriatr 2007;6:7-25.

48 Peters R, Beckett N, Forette F, Tuomilehto J, Clarke R, Ritchie C, et al. Incident dementia and blood pressure lowering in the Hypertension in the Very Elderly Trial cognitive function assessment (HYVET-COG): a double-blind, placebo controlled trial. Lancet Neurol 2008;7:683-9.

49 Li N-C, Lee A, Whitmer RA, Kivipelto M, Lawlor E, Kazis LE, et al. Use of angiotensin receptor blockers and risk of dementia in a predominantly male population: prospective cohort analysis. BMJ 2010;340:b5465.

50 Duron E, Hanon O. Vascular risk factors, cognitive decline, and dementia. Vasc Health Risk Manag 2008;4:363-81.

51 Stewart R, White LR, Xue QL, Launer LJ. Twenty-six-year change in total cholesterol levels and incident dementia: the Honolulu-Asia Aging Study. Arch Neurol 2007;64:103-7.

52 Murray CJL, Ezzati M, Lopez AD, Rodgers A, Vander Hoorn S. Comparative quantification of health risks: conceptual framework and methodological issues. Popul Health Metr 2003;1:1-20.

Accepted: 14 June 2010 